\title{
Phylogeny and Immunoreactivity of Norovirus GII.P16-GII.2, Japan, Winter 2016-17
}

\author{
Koo Nagasawa, ${ }^{1}$ Yuki Matsushima, ${ }^{1}$ \\ Takumi Motoya, Fuminori Mizukoshi, Yo Ueki, \\ Naomi Sakon, Koichi Murakami, \\ Tomomi Shimizu, Nobuhiko Okabe, \\ Noriko Nagata, Komei Shirabe, Hiroto Shinomiya, \\ Wataru Suzuki, Makoto Kuroda, \\ Tsuyoshi Sekizuka, Akihide Ryo, Kiyotaka Fujita, \\ Kazunori Oishi, Kazuhiko Katayama, \\ Hirokazu Kimura
}

During the 2016-17 winter season in Japan, human norovirus GII.P16-GIl.2 strains (2016 strains) caused large outbreaks of acute gastroenteritis. Phylogenetic analyses suggested that the 2016 strains derived from the GII.2 strains detected during 2010-12. Immunochromatography between 2016 strains and the pre-2016 Gll.2 strains showed similar reactivity.

Torovirus is a major cause of acute gastroenteritis in 1 humans and is genetically classified into 7 genogroups (GI-GVII). Among them, norovirus GI, GII, and GIV infect humans, and human norovirus (HuNoV) has many genotypes (1). GII viruses are common and have 22 confirmed genotypes (1).

In Infectious Diseases Weekly Report (https://www. niid.go.jp/niid/en/idwr-e.html), the Japanese national surveillance system reports the number of patients with acute

Author affiliations: National Institute of Infectious Diseases, Tokyo, Japan (K. Nagasawa, K. Murakami, M. Kuroda, T. Sekizuka, K. Oishi, H. Kimura); Kawasaki City Institute for Public Health, Kanagawa, Japan (Y. Matsushima, T. Shimizu, N. Okabe); Ibaraki Prefectural Institute of Public Health, Ibaraki, Japan (T. Motoya, N. Nagata); Tochigi Prefectural Institute of Public Health and Environmental Science, Tochigi, Japan (F. Mizukoshi); Miyagi Prefectural Institute of Public Health and Environment, Miyagi, Japan (Y. Ueki); Osaka Prefectural Institute of Public Health, Osaka, Japan (N. Sakon); Yamaguchi Prefectural Institute of Public Health and Environment, Yamaguchi, Japan (K. Shirabe); Ehime Prefectural Institute of Public Health and Environmental Science, Ehime, Japan (H. Shinomiya); Eiken Chemical Co. Ltd., Tochigi (W. Suzuki); Yokohama City University Graduate School of Medicine, Kanagawa (A. Ryo, H. Kimura); Gumma Paz University, Gunma, Japan (K. Fujita); Kitasato University, Minato-ku, Tokyo (K. Katayama)

DOI: https://doi.org/10.3201/eid2401.170284 gastroenteritis and their pathogenic agents who were examined at clinics or hospitals of the sentinel surveillance medical institutes $(\approx 3,000$ institutions $)$. This report indicated that HuNoV GII.2 was the third or fourth most prevalent genotype during the past 5 seasons (2011-2016), but became the predominant strain during the 2016-17 season. Of note, the total number of children with acute gastroenteritis in 2016-17 was the second largest over the past 11 epidemiologic seasons $(2,3)$.

On the basis of these observations, we used the full length of the RNA-dependent RNA polymerase (RdRp) region and capsid (VP1) gene to study the phylogeny of HuNoV strains detected during 2016-17 winter season (2016 strains). We also determined the immunoreactivities of the variant strains by various immunochromatography (IC) kits and the bioluminescent enzyme immunoassay (BLEIA).

\section{The Study}

In this study, we analyzed 26 GII.2 strains detected during October 2016-January 2017. Of those, we analyzed 19 strains for their phylogeny, and examined 7 strains by IC and BLEIA (4). We collected samples from the patients (children and adults) with acute gastroenteritis (mean \pm SD age $9.2 \pm$ 12.0 years). Because we could not collect adequate amounts of fecal specimens in some instances, we could not perform sequence analyses for all of them. Moreover, to compare the sensitivities of IC and BLEIA for the previous and current GII.P16-GII.2 strains, we conducted these tests using 2 fecal specimens containing GII.P16-GII.2 virus detected in 2009. These specimens, stored at $-80^{\circ} \mathrm{C}$, were obtained from clinics or hospitals, such as the sentinel surveillance medical institutions in Ibaraki Prefecture and Kawasaki City in Japan. We obtained written informed consent from the patients or their guardians for the samples. The study protocols were approved by the National Institute of Infectious Diseases for Public Health Ethics Committees (No. 576).

We performed viral RNA extraction and reverse transcription PCRs as described (5). To analyze the complete RdRp region and VP1 gene, we used primer-walking methods with primers designed by the PrimaClade server (online Technical Appendix Table 1, https://wwwnc.cdc.gov/EID/ article/24/1/17-0284-Techapp1.pdf) (6) and performed sequencing as described (5). To determine the genotype of

\footnotetext{
${ }^{1}$ These authors contributed equally to this article.
} 
the current virus, we used Norovirus Genotyping Tool version 1.0 (7). The accession numbers of the current strains are listed in Technical Appendix Table 2. To construct the phylogenetic tree, we collected complete sequences of the VP1 gene and RdRp region of HuNoV from GenBank (online Technical Appendix Table 2). We used 61 reference strains for phylogenic analysis of the VP1 gene and 70 strains for the RdRp region. We selected the best substitution models using the Bayesian information criterion (BIC) method by MEGA 6.0 (http://www.megasoftware. net (8). Using sequences from both regions, we inferred phylogenetic trees by the maximum-likelihood method as implemented in MEGA $6.0(8)$.
To explore the immunoreactivities of 2016 strains, we used 7 fecal specimens from the patients with acute gastroenteritis attributed to the 2016 strains to assess relationships among these strains' genome copy numbers and the sensitivities of 5 commercial IC kits: ImmunoCatch-Noro (Eiken Chemical, Tokyo, Japan); Quick Chaser-Noro (Mizuho Medy, Tosu-shi, Japan); Quick Navi-Noro 2 (Denka Seiken, Tokyo, Japan); GE test Noro Nissui (Nissui Pharmaceutical, Tokyo, Japan); RIDA QUICK Norovirus (R-Biopharm AG, Darmstadt, Germany). In addition, we evaluated the BLEIA (Eiken Chemical, Tokyo) to compare sensitivities for the IC kits to the 2016 strains. We quantified HuNoV genome numbers by using a real-time PCR (9). Furthermore, to test the

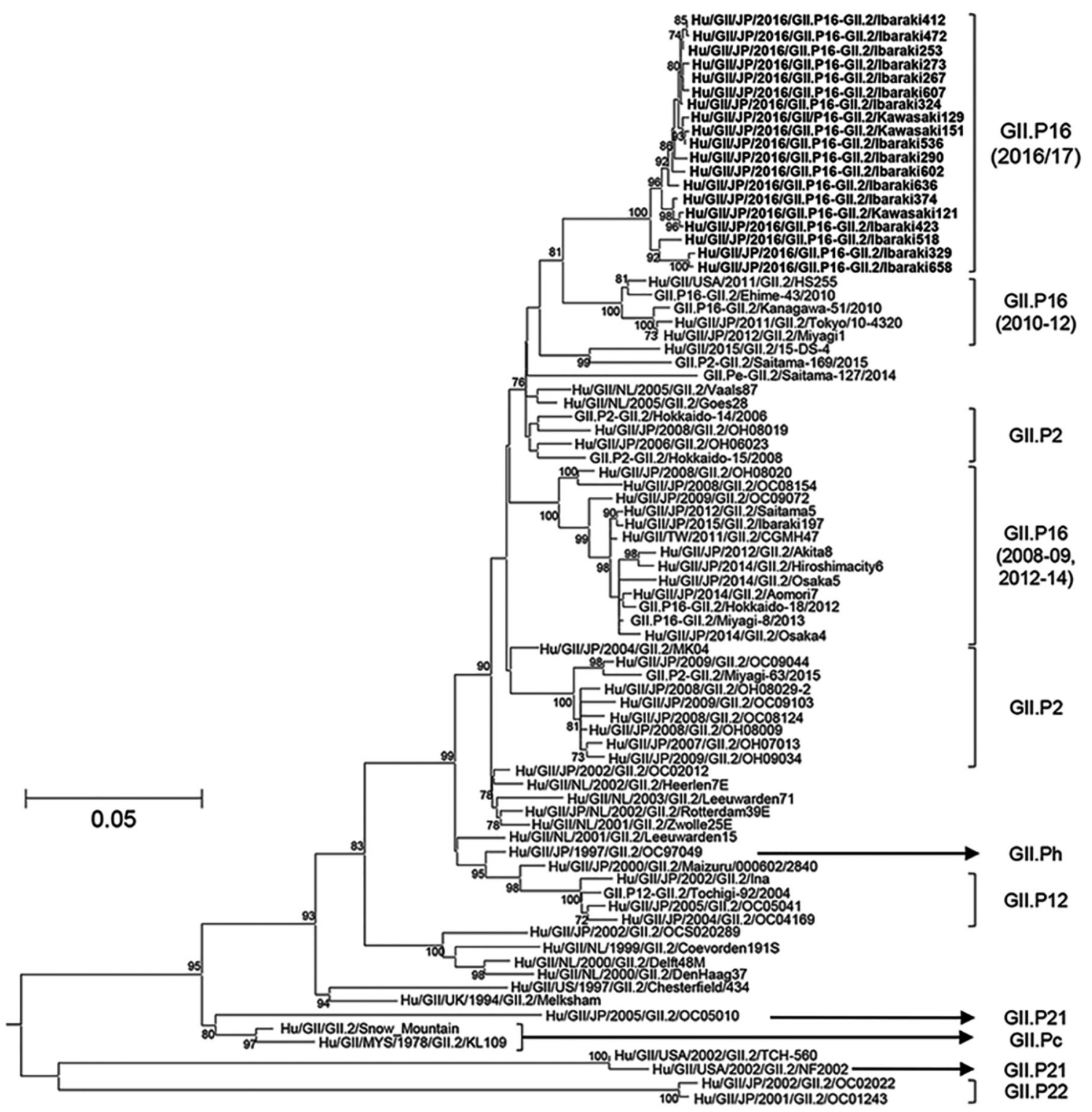

Figure 1. Phylogenetic tree for the capsid (VP1) gene in human norovirus GII strains. The tree was constructed by using the maximumlikelihood method. Bold letters denote GII.2v strains. Numbers at branch nodes show bootstrap values with $\geq 70 \%$ support. Scale bar represents number of nucleotide substitutions per site. 
sensitivity of the IC kits and BLEIA with the GII.2 strains before and during the 2016-2017 winter season, we used 2 fecal specimens collected in 2009-10.

The genotype of HuNoV detected in the 2016-17 season was GII.P16-GII.2. The maximum-likelihood tree of the VP1 genes formed many clusters (Figure 1). The 2016 strains diverged from common ancestors of the GII. P16-GII.2 cluster detected in 2010-12. The phylogenetic tree of the RdRp coding region also formed many clusters
(Figure 2). The common ancestors of GII.P16 strains diverged into 2 clusters; one contains strains with capsid genotypes GII.3, GII.4, GII.13, and GII.2 detected in 2010-12, and the other one contains strains with capsid genotypes GII.17 and GII.2 detected in 2009-10 and 2012-15. On the basis of these trees, the 2016 strains could have diverged from GII.2 detected in 2010-12. Finally, RdRp of the 2016 strains diverged from the common ancestors of the 2016 strains and GIIP16-GII.4.

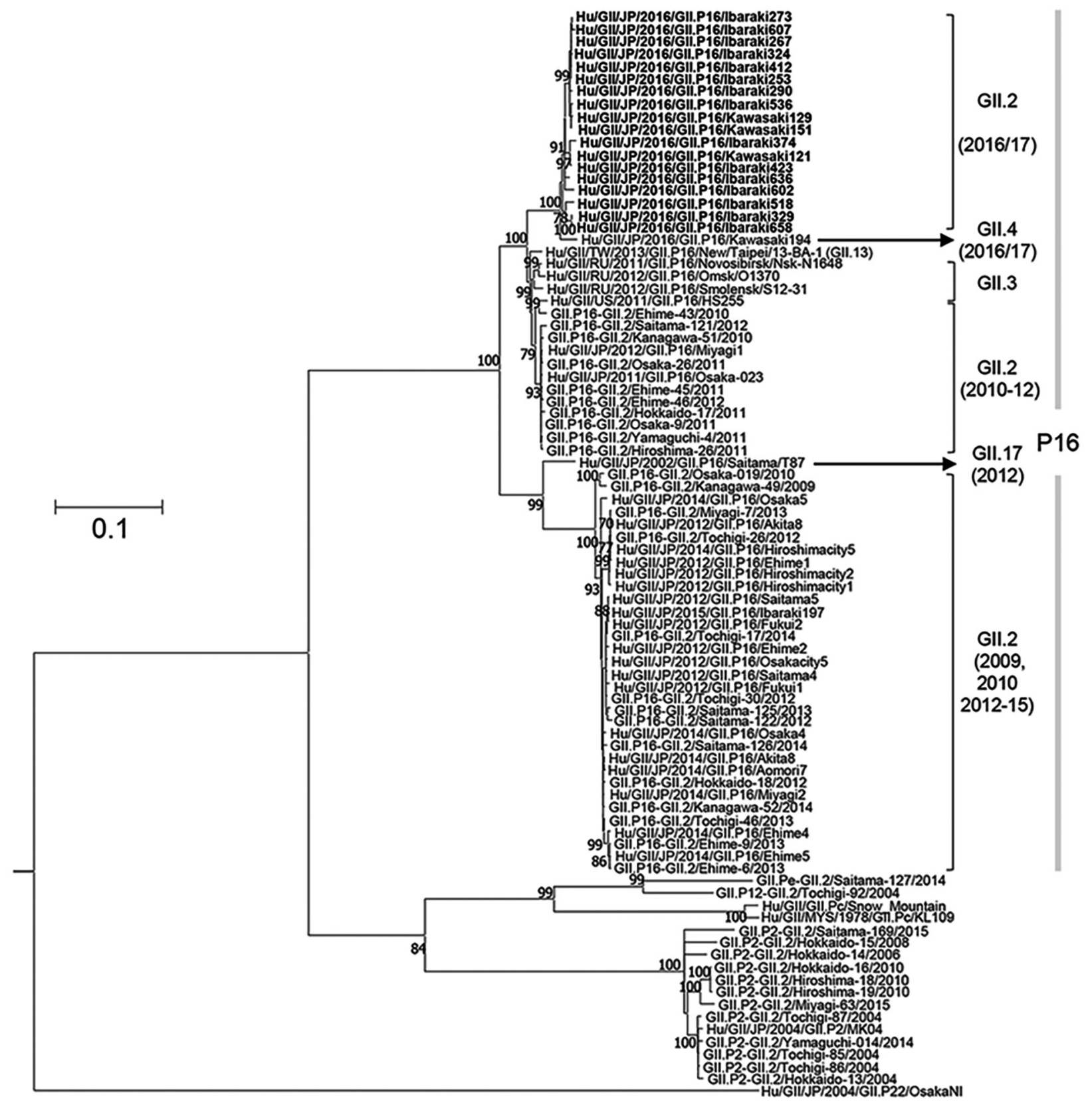

Figure 2. Phylogenetic tree for the RNA-dependent RNA polymerase (RdRp) region in human norovirus GII strains. The tree was constructed by using the maximum-likelihood method. Bold letters denote GII.2v strains. Numbers at branch nodes show bootstrap values with $\geq 70 \%$ support. Scale bar represents number of nucleotide substitutions per site. 
Phylogeny and Immunoreactivity of NoV 2016 Strains

Table. Sensitivities of 5 immunochromatography kits and the BLEIA for 2016 and pre-2016 GII.P16-GII2 norovirus strains*

\begin{tabular}{|c|c|c|c|c|c|c|c|}
\hline \multirow[b]{2}{*}{ Sample ID } & \multirow{2}{*}{$\begin{array}{c}\text { Virus } \\
\text { genome, } \\
\text { copies/g }\end{array}$} & \multicolumn{5}{|c|}{ Norovirus immunochromatography kit test results } & \multirow[b]{2}{*}{$\begin{array}{l}\text { BLEIA } \\
\text { result }\end{array}$} \\
\hline & & $\begin{array}{c}\text { ImmunoCatch- } \\
\text { Noro }\end{array}$ & $\begin{array}{c}\text { Quick } \\
\text { Chaser-Noro }\end{array}$ & $\begin{array}{l}\text { Quick Navi- } \\
\text { Noro } 2\end{array}$ & $\begin{array}{c}\text { GE test } \\
\text { Noro Nissui }\end{array}$ & $\begin{array}{l}\text { RIDA } \\
\text { QUICK }\end{array}$ & \\
\hline Kawasaki181, 2016 strains & $9.12 \times 10^{9}$ & + & + & + & + & + & + \\
\hline Kawasaki159, 2016 strains & $7.47 \times 10^{8}$ & + & + & + & - & + & + \\
\hline Kawasaki163, 2016 strains & $2.30 \times 10^{7}$ & + & + & - & - & + & + \\
\hline Kawasaki173, 2016 strains & $1.31 \times 10^{7}$ & + & - & - & - & - & + \\
\hline Kawasaki125, 2016 strains & $2.26 \times 10^{6}$ & - & - & - & - & - & + \\
\hline Kawasaki175, 2016 strains & $1.82 \times 10^{6}$ & - & - & - & - & - & - \\
\hline Kawasaki177, 2016 strains & $3.16 \times 10^{5}$ & - & - & - & - & - & - \\
\hline Ibaraki09-1095, pre-2016 GII.P16-GII.2 & $8.09 \times 10^{7}$ & + & - & - & - & - & + \\
\hline Ibaraki09-965, pre-2016 GII.P16-GII.2 & $1.40 \times 10^{7}$ & + & - & - & - & - & + \\
\hline
\end{tabular}

To examine the antigenicity between 2016 strains and pre-2016 GII.2 strains, we assessed the relationship between the quantities of GII.2 genome and the results of the IC kits and BLEIA (Table). The IC kits we used in this study all showed the 2016 strains positive, when $\approx 10^{10}$ copies/g of viral genome were in each sample. The reactivities of these kits against lower copy numbers of 2016 strains and pre-2016 GII.2 strains were significantly different. For example, 1 kit (ImmunoCatch-Noro) was $>100$ times more sensitive than another kit (GE test Noro Nissui) used in this study. BLEIA could detect samples with 2016 strains of genome copy number of $\approx 2.3 \times 10^{6}$ copies $/ \mathrm{g}$ in fecal samples.

\section{Conclusions}

We describe the phylogeny and immunoreactivity of the strains that suddenly emerged in Japan in 2016. As shown in the trees, the 2016 strains diverged from the HuNoV GII.P16-GII.2 detected in 2010-12, rather than that detected in 2015. Tohma et al. showed that the GII.P16 strains circulating in the 2016-17 winter season carried 4 amino acid mutations (S293T, V332I, $\mathrm{K} 357 \mathrm{Q}$, and $\mathrm{T} 360 \mathrm{~A})$ in their polymerase from pre2016 GII.P16-GII.2 strains (10). Among them, all 2016 strains had amino acid mutations (S293T, K357Q, and T360A), and 17 of the 19 strains also had the V332I mutation. Moreover, some reports suggested that the GII. P16-GII.2 strains detected in 2016 were a new recombinant strain, and both the RdRp region and VP1 gene sequences of our strains and the new strains were similar (identity $\approx 98 \%-99 \%$; data not shown), although the analyzed sequence lengths were different $(11,12)$. However, in this study, we could not obtain clear data for the recombinant strains.

Previous reports showed that IC kits were positive for most of the HuNoV GII genotypes in clinical samples (fecal specimens), corresponding to around $10^{7}$ copies of norovirus genome per gram in the fecal specimen $(13,14)$. In contrast, another report showed that the reactivity of the pre-2016 GII.2 strains was lower than other genotypes (15). We obtained similar data on the sensitivity in each kit between the pre-2016 GII.P16GII.2 and 2016 strains. However, we saw substantially different sensitivities among these IC kits (maximum 100 -fold). Our findings show that the IC kits may be valuable for detecting the GII.P16-GII.2 strains, including pre-2016 GII.2 and 2016 strains, on a case-by-case basis or as a backup test in the laboratories with a rapid bedside norovirus diagnosis.

The 2016 NoV strains caused outbreaks of acute gastroenteritis in many countries $(2,10-12)$. Additional molecular epidemiologic analyses are needed to track the epidemics and to better understand the viral evolution.

\section{Acknowledgments}

We thank Gary C. Howard and Yoshiyuki Suzuki for a critical review of this manuscript.

This work was partly supported by a commissioned project for Research on Emerging and Re-emerging Infectious Diseases from Japan's Agency for Medical Research and Development.

Dr. Nagasawa is a pediatrician and a researcher at the National Institute of Infectious Diseases. His research interests are viral infectious diseases and molecular evolution of respiratory and gastroenteric virus. Mr. Matsushima is a pharmacist and a researcher at Kawasaki City Institute for Public Health, Kanagawa, Japan. His research interest is the molecular epidemiology of viral infectious diseases in humans.

\section{References}

1. Vinjé J. Advances in laboratory methods for detection and typing of norovirus. J Clin Microbiol. 2015;53:373-81. http://dx.doi.org/10.1128/JCM.01535-14

2. Thongprachum A, Okitsu S, Khamrin P, Maneekarn N, Hayakawa S, Ushijima H. Emergence of norovirus GII.2 and its novel recombination during the gastroenteritis outbreak in Japanese children in mid-2016. Infect Genet Evol. 2017;51:86-8. http://dx.doi.org/10.1016/j.meegid.2017.03.020

3. National Institute of Infectious Diseases. Japan. Infectious gastroenteritis, 2017 [in Japanese] [cited 2017 Feb 16] 
http://www.nih.go.jp/niid/ja/10/2096-weeklygraph/ 1647-04gastro.html

4. Suzuki W, Ohiro Y, Tsukagoshi H, Kimura H. Evaluation of norovirus detection method based on a newly developed bioluminescent enzyme immunoassay (BLEIA) system [in Japanese]. Kansenshogaku Zasshi. 2015;89:230-6. http://dx.doi.org/10.11150/kansenshogakuzasshi.89.230

5. Kojima S, Kageyama T, Fukushi S, Hoshino FB, Shinohara M, Uchida K, et al. Genogroup-specific PCR primers for detection of Norwalk-like viruses. J Virol Methods. 2002;100:107-14 http://dx.doi.org/10.1016/S0166-0934(01)00404-9

6. Gadberry MD, Malcomber ST, Doust AN, Kellogg EA. Primaclade - a flexible tool to find conserved PCR primers across multiple species. Bioinformatics. 2005;21:1263-4. http://dx.doi.org/10.1093/bioinformatics/bti134

7. Kroneman A, Vennema H, Deforche K, v d Avoort H, Peñaranda $\mathrm{S}$, Oberste MS, et al. An automated genotyping tool for enteroviruses and noroviruses. J Clin Virol. 2011;51:121-5. http://dx.doi.org/10.1016/j.jcv.2011.03.006

8. Tamura K, Stecher G, Peterson D, Filipski A, Kumar S. MEGA6: Molecular Evolutionary Genetics Analysis version 6.0. Mol Biol Evol. 2013;30:2725-9. http://dx.doi.org/10.1093/ molbev/mst197

9. Kageyama T, Kojima S, Shinohara M, Uchida K, Fukushi S, Hoshino FB, et al. Broadly reactive and highly sensitive assay for Norwalk-like viruses based on real-time quantitative reverse transcription-PCR. J Clin Microbiol. 2003;41:1548-57. http://dx.doi.org/10.1128/JCM.41.4.1548-1557.2003

10. Tohma K, Lepore CJ, Ford-Siltz LA, Parra GI. Phylogenetic analyses suggest that factors other than the capsid protein play a role in the epidemic potential of GII. 2 norovirus. $\mathrm{mSphere}$. 2017;2:e00187-17. http://msphere.asm.org/content/2/3/e00187-17.

11. Lu J, Fang L, Sun L, Zeng H, Li Y, Zheng H, et al. Association of GII.P16-GII.2 recombinant norovirus strain with increased norovirus outbreaks, Guangdong, China, 2016. Emerg Infect Dis. 2017;23:1188-90. http://dx.doi.org/10.3201/eid2307.170333

12. Bidalot M, Théry L, Kaplon J, De Rougemont A, Ambert-Balay K. Emergence of new recombinant noroviruses GII.p16-GII.4 and GII. p16-GII.2, France, winter 2016 to 2017. Euro Surveill. 2017; 22:30508. http://dx.doi.org/10.2807/1560-7917.ES.2017. 22.15.30508

13. Takanashi S, Okame M, Shiota T, Takagi M, Yagyu F, Tung PG, et al. Development of a rapid immunochromatographic test for noroviruses genogroups I and II. J Virol Methods. 2008;148:1-8. http://dx.doi.org/10.1016/j.jviromet.2007.10.010

14. Bruggink LD, Witlox KJ, Sameer R, Catton MG, Marshall JA. Evaluation of the RIDA( $($ ) QUICK immunochromatographic norovirus detection assay using specimens from Australian gastroenteritis incidents. J Virol Methods. 2011;173:121-6. http://dx.doi.org/10.1016/j.jviromet.2011.01.017

15. Bruggink LD, Catton MG, Marshall JA. Evaluation of the Bioline Standard Diagnostics SD immunochromatographic norovirus detection kit using fecal specimens from Australian gastroenteritis incidents. Diagn Microbiol Infect Dis. 2013;76:147-52 http://dx.doi.org/10.1016/j.diagmicrobio.2013.02.018

Address for correspondence: Hirokazu Kimura, Infectious Disease Surveillance Center, National Institute of Infectious Diseases, 4-7-1 Gakuen, Musashimurayama-shi, Tokyo 208-0011, Japan; email: kimhiro@nih.go.jp or Kazuhiko Katayama, Laboratory of Viral Infection I, Kitasato Institute for Life Sciences Graduate School of Infection Control Sciences, Kitasato University, 5-9-1 Shirogane, Minato-ku, Tokyo 108-8641, Japan; email: katayama@lisci.kitasato-u.ac.jp

\section{EID Podcast:} Emerging Infectious Diseases Cover Art Byron Breedlove, managing editor of the journal, elaborates on aesthetic considerations and historical factors, as well as the complexities of obtaining artwork for Emerging Infectious Diseases.
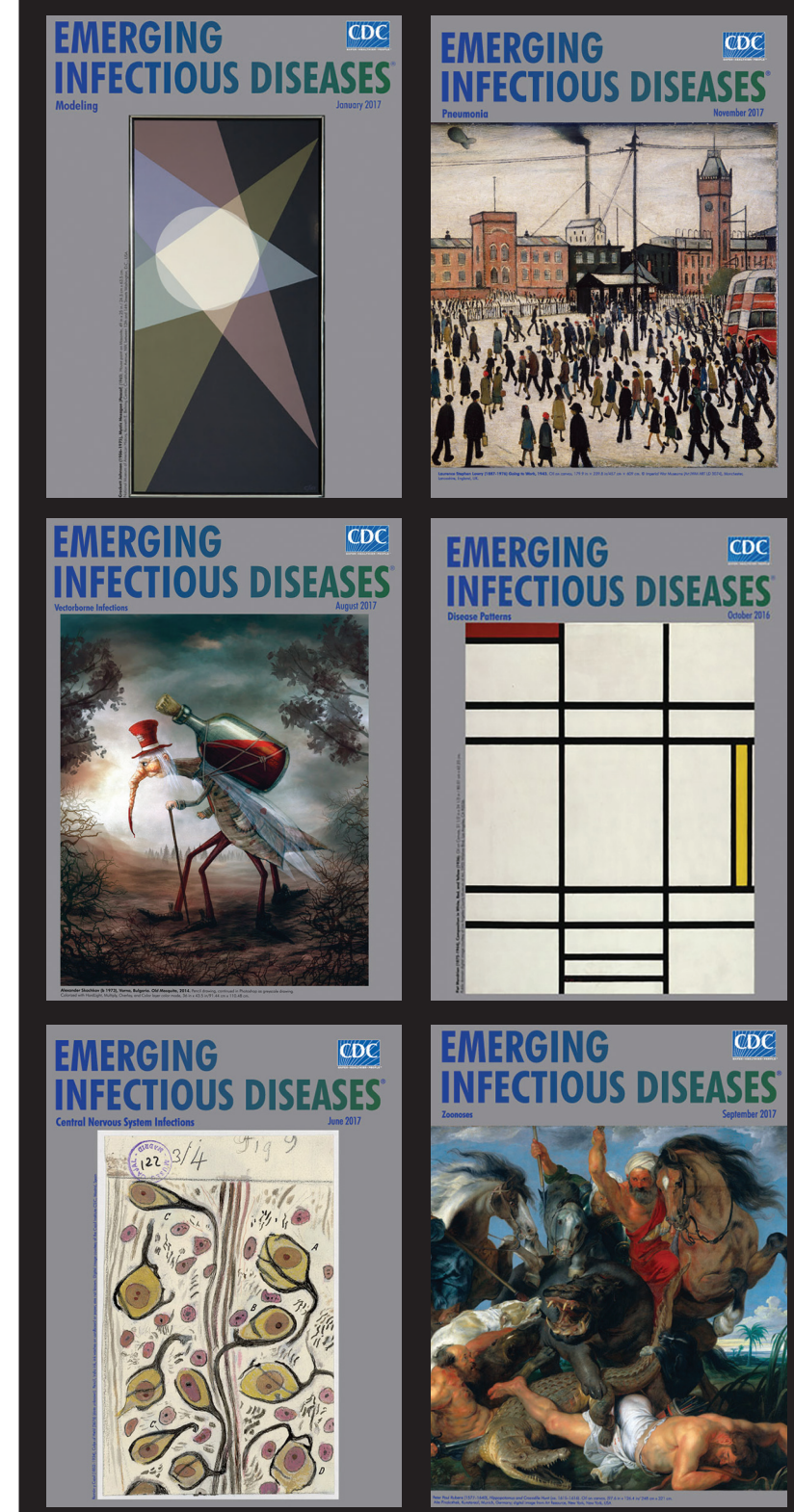

Visit our website to listen:

EMERCING INFECIIOUS DISEASES https://www2c.cdc.gov/ podcasts/player. asp?f $=\mathbf{8 6 4 6 2 2 4}$ 